\title{
Introduction to Gyrator Transform
}

\author{
T. Alieva, J. A. Rodrigo and M. L. Calvo \\ Universidad Complutense de Madrid, \\ Facultad de Ciencias Fisicas, Ciudad Universitaria s/n, \\ Madrid 28040, Spain
}

\begin{abstract}
We introduce the gyrator transform which produces a rotation of two-dimensional signal in phase space. Main properties of this transform are considered. The flexible optical set up for gyrator transform experimental implementation is discussed. The applications of the gyrator transform to image processing such as filtering, noise reduction, encryption are demonstrated..
\end{abstract}

Keywords: Fourier transforms, Optical systems, Optical image processing.

PACS: R42.30Kq, $42.15 \mathrm{Eq}$

\section{INTRODUCTION}

More than forty years ago, Anthony Van der Lugt introduced an optical scheme for correlation operation, based on a cascade of two first order optical system performing the Fourier transform, initiating an era of Fourier Optics [1]. Nowadays the Fourier Optics area has expanded with more sophisticated tools for signal processing such as wavelets, bilinear distributions, fractional transformations, etc. Nevertheless the firstorder optical systems, mathematically described by canonical integral transforms, remain the basic elements for analogue optical information processing. Several new applications of the first-order optical systems for information processing have been proposed in the past decade. In particular first-order optical systems, performing fractional Fourier transform, have been used for shift-variant filtering, noise reduction, encryption, etc [2]. Another ones have served as mode converters which permit to obtain the helicoidal vortex Laguerre-Gaussian mode or other stable modes carrying the fractional topological charge [3] after the propagation of the Hermite-Gaussian beam through them $[3,4]$. The generalized mode converter can be described by the gyrator transform (called in [5] as a cross-gyrator), which as well as the fractional Fourier transform belongs to the class of the linear canonical integral transforms. In the contrast to the fractional Fourier transform (see for example [2] and references there in), the gyrator operation is still little known for the optical community. In order to include the gyrator transform in the list of image processing tools we have to study its properties and to design an optimal scheme for its optical implementation that will be discussed in this contribution. We will also propose several applications of this transform for information processing.

CP949, $6^{\text {th }}$ International Workshop on Information Optics (WIO '07)

edited by J. A. Benediktsson, B. Javidi, K. S. Gudmundsson

(C) 2007 American Institute of Physics 978-0-7354-0463-2/07/\$23.00 


\section{DEFINITION AND PROPERTIES OF THE GYRATOR TRANSFORM}

Gyrator operation is mathematically defined as a linear canonical integral transform which produces the rotation in twisted position-spatial frequency planes $\left(x, q_{y}\right)$ and $\left.\left(y, q_{x}\right\}\right)$ of phase space [5]. Thus the gyrator transform (GT) at parameter $\alpha$, called as a rotation angle, of a two-dimensional function $f_{i}\left(x_{i}, y_{i}\right)$ can be written in the following form

$$
\begin{aligned}
f_{o}\left(\mathbf{r}_{o}\right) & =R^{\alpha}\left[f_{i}\left(\mathbf{r}_{i}\right)\right]\left(\mathbf{r}_{o}\right)=\iint f_{i}\left(x_{i}, y_{i}\right) K_{\alpha}\left(x_{i}, y_{i}, x_{o}, y_{o}\right) d x_{i} d y_{i} \\
& =\frac{1}{|\sin \alpha|} \iint f_{i}\left(x_{i}, y_{i}\right) \exp \left(i 2 \pi \frac{\left(x_{o} y_{o}+x_{i} y_{i}\right) \cos \alpha-\left(x_{i} y_{o}+x_{o} y_{i}\right)}{\sin \alpha}\right) d x_{i} d y_{i},
\end{aligned}
$$

For $\alpha=0$ it corresponds to the identity transform, for $\alpha=\pi / 2$ it reduces to the directinverse Fourier transform with rotation of the coordinates at $\pi / 2$, for $\alpha=\pi$ the reverse transform is obtained. The GT is periodic and additive with respect to parameter $\alpha: R^{\alpha}$ $R^{\beta}=R^{\alpha+\beta}$. The inverse GT corresponds to the GT at angle $-\alpha$.

The main theorems for the GT such as scaling, shift, modulation, Parseval relation, etc. have been derived in [7]. They are displayed in Table 1, where we use the following notations for the vectors

$$
\mathbf{r}^{t}=(x, y) ; \mathbf{v}^{t}=\left(v_{x}, v_{y}\right), \overrightarrow{\mathbf{v}}^{t}=\left(v_{y}, v_{x}\right) ; \mathbf{k}^{t}=\left(k_{x}, k_{y}\right), \hat{\mathbf{k}}^{t}=\left(k_{y}, k_{x}\right) .
$$

The parameters $\sigma_{\alpha}, \sigma_{\beta}, \mathbf{S}, \beta$ are defined as

$$
\sigma_{\alpha}=\operatorname{sgn}(\sin \alpha), \sigma_{\beta}=\operatorname{sgn}(\sin \beta), \mathbf{S}=\left(\begin{array}{cc}
s_{x} & 0 \\
0 & s_{y}
\end{array}\right), \cot \beta=\frac{\cot \alpha}{s_{x} s_{y}} .
$$

\begin{tabular}{|c|c|}
\hline Shift theorem & $\begin{aligned} R^{\alpha}\left[f_{i}\left(\mathbf{r}_{i}-\mathbf{v}\right)\right]\left(\mathbf{r}_{o}\right)= & \exp \left(i \pi\left(v_{x} v_{y} \sin 2 \alpha-2 \mathbf{r}_{o}^{t} \mathbf{v} \sin \alpha\right)\right) \\
& \times R^{\alpha}\left[f_{i}\left(\mathbf{r}_{i}\right)\right]\left(\mathbf{r}_{o}-\mathbf{v} \cos \alpha\right)\end{aligned}$ \\
\hline Plane wave modulation & $\begin{aligned} R^{\alpha}\left[f_{i}\left(\mathbf{r}_{i}\right) \exp \left(-i 2 \pi \mathbf{k}^{t} \mathbf{r}_{i}\right)\right]\left(\mathbf{r}_{o}\right)= & \exp \left(-i \pi\left(k_{x} k_{y} \sin 2 \alpha\right)\right) \\
& \times \exp \left(-i \pi 2 \mathbf{k}^{y} \mathbf{r}_{o} \cos \alpha\right) \\
& \times R^{\alpha}\left[f_{i}\left(\mathbf{r}_{i}\right)\right]\left(\mathbf{r}_{o}+\hat{\mathbf{k}} \sin \alpha\right)\end{aligned}$ \\
\hline Scaling theorem & $\begin{aligned} R^{\alpha}\left[f_{i}\left(\mathbf{S r}_{i}\right)\right]\left(\mathbf{r}_{o}\right) & =\frac{\sigma_{\beta} \cos \beta}{\sigma_{\alpha} \cos \alpha} \exp \left(i 2 \pi x_{o} y_{o}\left(1-\left(\frac{\cos \beta}{\cos \alpha}\right)^{2}\right) \cot \alpha\right) \\
& \times R^{\beta}\left[f_{i}\left(\mathbf{r}_{i}\right)\right]\left(\frac{\cos \beta}{\cos \alpha} \mathbf{S r}_{o}\right)\end{aligned}$ \\
\hline Parseval relation & $\left\lceil R^{\alpha}\left[f_{i}\left(\mathbf{r}_{i}\right)\right]\left(\mathbf{r}_{o}\right)\left(R^{\alpha}\left[g_{i}\left(\mathbf{r}_{e}\right)\right]\left(\mathbf{r}_{o}\right)\right)^{*} d \mathbf{r}_{o}=\left\lceil f_{i}\left(\mathbf{r}_{i}\right) g_{i}\left(\mathbf{r}_{i}\right)^{*} d \mathbf{r}_{i}\right.\right.$ \\
\hline
\end{tabular}

TABLE 1. Main properties of the gyrator transform. 
The analytical expressions for the GT of basic functions such as Dirac delta, hyperbolic, plane and spherical waves, Gaussian mode have been recently calculated [7] and are summarized in Table 2.

TABLE 2. Gyrator transform of selected functions.

\begin{tabular}{|l|l|}
\hline$f_{i}\left(\mathbf{r}_{i}\right)$ & $f_{o}\left(\mathbf{r}_{o}\right)=R^{\alpha}\left[f_{i}\left(\mathbf{r}_{i}\right)\right]\left(\mathbf{r}_{o}\right)$ \\
\hline$\delta\left(\mathbf{r}_{i}-\mathbf{v}\right)$ & $\frac{1}{|\sin \alpha|} \exp \left(i 2 \pi \frac{\left(x_{o} y_{o}+v_{x} v_{y}\right) \cos \alpha-\left(v_{x} y_{o}+x_{o} y_{y}\right)}{\sin \alpha}\right)$ \\
\hline $\exp \left(i 2 \pi c x_{i} y_{i}\right)$ & $\frac{1}{|\sin \alpha|} \exp \left(i 2 \pi \frac{c \cot \alpha-1}{c+\cot \alpha} x_{o} y_{o}\right),(c \neq-\cot \alpha)$ \\
\hline $\exp \left(-i \mathbf{k}^{t} \mathbf{r}_{i}\right)$ & $\frac{1}{|\sin \alpha|} \exp \left(-i 2 \pi\left(x_{o} y_{o}+k_{x} k_{y}\right) \tan \alpha\right) \exp \left(-\frac{i}{\cos \alpha} \mathbf{k}^{t} \mathbf{r}_{o}\right)$ \\
\hline $\exp \left(-i \pi b \mathbf{r}_{i}^{2}\right)$ & $\frac{1}{\sqrt{\cos ^{2} \alpha-b^{2} \sin ^{2} \alpha}} \exp \left(-i \pi \frac{\left(1+b^{2}\right) \sin 2 \alpha}{\cos ^{2} \alpha-b^{2} \sin ^{2} \alpha} x_{o} y_{o}\right) \exp \left(\frac{-i \pi b \mathbf{r}_{o}^{2}}{\cos ^{2} \alpha-b^{2} \sin ^{2} \alpha}\right)$ \\
\hline $\exp \left(-\pi a \mathbf{r}_{i}^{2}\right)$ & $\frac{1}{\sqrt{\cos ^{2} \alpha+a^{2} \sin ^{2} \alpha}} \exp \left(i \pi \frac{\left(a^{2}-1\right) \sin _{2} 2 \alpha}{\cos ^{2} \alpha+a^{2} \sin ^{2} \alpha} x_{o} y_{o}\right) \exp \left(\frac{-\pi a \mathbf{r}_{o}^{2}}{\cos ^{2} \alpha+a^{2} \sin ^{2} \alpha}\right)$ \\
\hline
\end{tabular}

Note that the GT for angle $\alpha=(\pi(2 k+1) / 4$ with integer $k$ transforms the Hermite Gaussian mode $H G_{m n}$ into Laguerre Gaussian one, which carries the orbital angular momentum if $m * n$.

Moreover the Hermite Gaussian modes rotated at angle $\pi(2 k+1) / 4$ are eigenfunctions for the GT for any $\alpha$.

\section{EXPERIMENTAL SET UP FOR GYRATOR TRANSFORM}

Based on the matrix formalism for first-order lossless optical systems, it has been recently shown [6] that the GT for the large range of angles can be realized by a flexible optical system which contains three generalized lenses with fixed distance between them. The angle $\alpha$ is changed by rotation of the cylindrical lenses which form the generalized lenses. Other possibility is to use the spatial light modulator for variable lens performance.

The simplest flexible set up performing the GT consists from the three generalized lenses $L_{1}, L_{2}$ and $L_{3}$. The lenses $L_{1}$ and $L_{3}$ are identical. Every generalized lens is a superposition of two convergent cylindrical ones rotated at the angles $\phi_{1}=-\varphi$ and $\phi_{2}$ $=\varphi-\pi / 2$ with respect to the vertical axis $O Y$, correspondingly. The action of a generalized lens at the complex field amplitude $g_{i}$ leads to the quadratic phase modulation written as

$$
g_{o}\left(x_{o}, y_{o}\right)=\exp \left(-i \pi \frac{x_{o}^{2}+y_{o}^{2}-2 x_{o} y_{o} \sin 2 \varphi}{\lambda f}\right) g_{i}\left(x_{o}, y_{o}\right),
$$


where $\lambda$ is the wavelength and $f$ is the focal distance of a cylindrical lens. If the distances between the generalized lenses are equal, denoted here by $z$, which is related to the focal distances of the lenses as $f_{1}=f_{3}=2 f_{2}=z$ and

$$
\begin{aligned}
\sin 2 \varphi_{1} & =\sin 2 \varphi_{3}=\cot (\alpha / 2), \\
\sin 2 \varphi_{2} & =(\sin \alpha) / 2
\end{aligned}
$$

then the complex field amplitude at the output of the system is the GT of the one at the input plane

$$
f_{o}\left(\mathbf{r}_{o}\right)=-\frac{1}{\lambda z|\sin \alpha|} \iint f_{i}\left(x_{i}, y_{i}\right) \exp \left(i 2 \pi \frac{\left(x_{o} y_{o}+x_{i} y_{i}\right) \cos \alpha-\left(x_{i} y_{o}+x_{o} y_{i}\right)}{\lambda z \sin \alpha}\right) d x_{i} d y_{i},(6)
$$

where $\lambda z$ is a scaling parameter. It is easy to see from the last relation that this set up is able to perform the GT only for the angles $\alpha \in[\pi / 2,3 \pi / 2]$. Nevertheless since

$$
R^{\alpha+\pi}\left[f_{i}\left(\mathbf{r}_{i}\right)\right]\left(\mathbf{r}_{o}\right)=R^{\alpha}\left[f_{i}\left(\mathbf{r}_{i}\right)\right]\left(-\mathbf{r}_{o}\right)
$$

we can cover entire interval $\alpha \in[0,2 \pi]$ if it is necessary.

We observe that for $\alpha=\pi$, that happens when the generalized lenses $L_{1}, L_{2}$ and $L_{3}$ are reduced to the spherical lenses, the common 4- $f$ system - a cascade of two Fourier transforming systems - is obtained. Therefore the proposed gyrator system can be considered as a generalization of the well known 4- $f$ system to the case of the generalized lenses.

\section{APPLICATIONS OF THE GYRATOR TRANSFORM FOR IMAGE PROCESSING}

The possible applications of the gyrator transform for image processing are similar to the ones proposed for the symmetric fractional Fourier transform [2, 8 and references there in]. This conclusion is based on the facts that image is a positive function and that the gyrator transform can be represented as a rotated at angle $-\pi / 4$ antisymmetric fractional FT of the original image rotated at $\pi / 4$ [9]. Here we will discuss the applications of the GT for image filtering, pattern recognition, noise reduction and encryption. The test image is displayed in Fig. 1.

In optical signal processing the phase only filters are playing an important role due to its high efficiency comparing with the amplitude ones. Therefore let us consider the possibility of application of phase only filters in the gyrator domain. We will analyse the quality of reconstruction of the test image from only phase or only amplitude of its GT for different angles $\alpha$. Thus if we introduce the following notation

$$
R^{\alpha}\left[f_{i}\left(\mathbf{r}_{i}\right)\right]\left(\mathbf{r}_{o}\right)=F_{\alpha}\left(\mathbf{r}_{o}\right) \exp \left(i \varphi_{\alpha}\left(\mathbf{r}_{o}\right)\right),
$$


where $F_{\alpha}>0$ is the amplitude, and $\varphi_{\alpha}$ is the phase of the GT at angle $\alpha$ the operations we perform are expressed as $R^{-\alpha}\left[\exp \left(i \varphi_{\alpha}\left(\mathbf{r}_{o}\right)\right)\right](\mathbf{r})$ and $R^{-\alpha}\left[F_{\alpha}\left(\mathbf{r}_{o}\right)\right](\mathbf{r})$. In Fig. 2 the amplitude of the image reconstructed from the phase-only data $(a, b, c)$ and the amplitude-only data (d, e, f) of the GT of the test image are displayed for different values of the transformation angle $\alpha=10^{\circ}(\mathrm{a}, \mathrm{d}), 60^{\circ}(\mathrm{b}, \mathrm{e}), 90^{\circ}(\mathrm{c}, \mathrm{f})$.

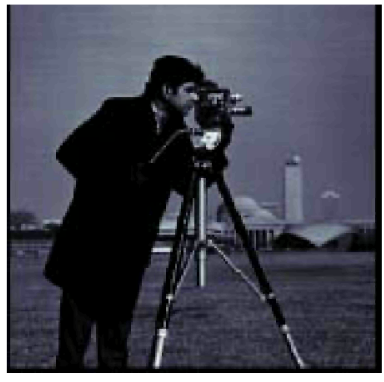

FIGURE 1. Test image.

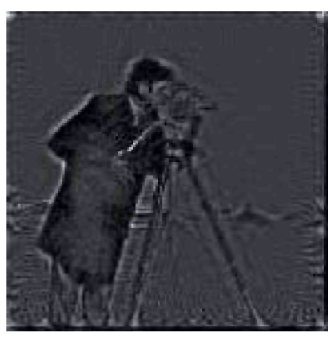

a

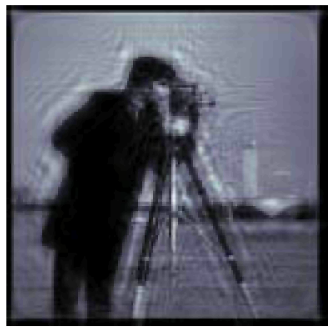

d

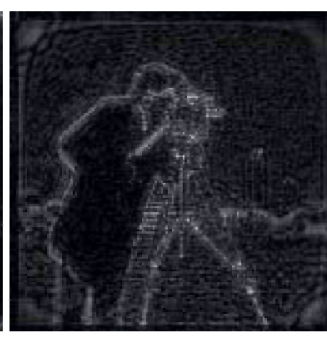

b

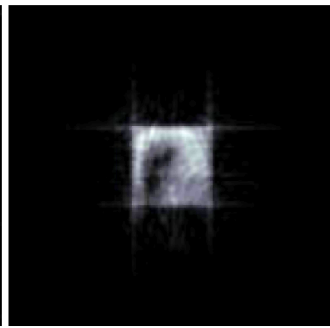

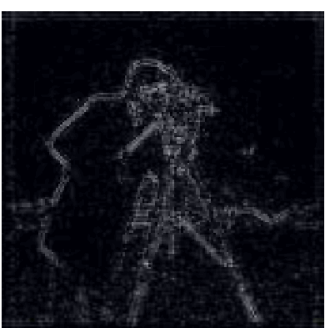

$\mathrm{c}$

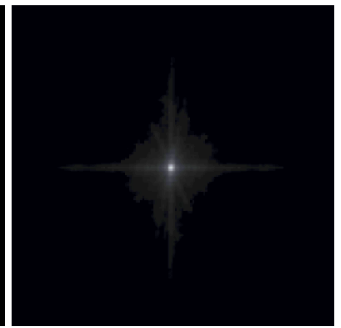

f

FIGURE 2. The image reconstruction from the phase-only $(\mathrm{a}, \mathrm{b}, \mathrm{c})$ and amplitude-only $(\mathrm{d}, \mathrm{e}, \mathrm{f})$ data of the GT for $\alpha=10^{\circ}(\mathrm{a}, \mathrm{d}), 60^{\circ}(\mathrm{b}, \mathrm{e}), 90^{\circ}(\mathrm{c}, \mathrm{f})$.

We observe that the amplitude of the GT contains essential information about the image only for relatively small $\alpha$ meanwhile the GT phase preserves the information about the image edges almost for all parameter range. It means that the phase-only filters can be successfully implemented in any gyrator domain. They can be used for 
example for shift variant pattern recognition. Indeed as it follows from the shift theorem (see Table 1) that the correlation operation performed in the gyrator domain is shift variant. As well as the in the case of the fractional FT [10] the pattern size and the parameter $\alpha$ control the region where the pattern can be detected.

Let us consider the effect of application of the same filter in the different gyrator domains. We have chosen two circular binary amplitude filters: H-filter (opaque circle on the transparent background) and L-filter (transparent circle on the opaque background). They correspond to high pass $(\mathrm{H})$ and low pass (L) filters if are located in the Fourier domain ( $\alpha=\pi / 2)$. First of all in Figs. 3 - 4 we observe that filtering is not shift invariant if angle $\alpha * \pi / 2$. The application of the H-filter in the GT domain (see Fig. 3) leads to the local high frequency pass filtering, where the area of its action depends on the filter size and the parameter $\alpha$. In particular such type of filters can be used for selective edge enhancement. The L-filter (see Fig. 4) produces image cutting together with smoothing. The area of image reproduction and the degree of smoothing are enlarged with increasing of $\alpha$.
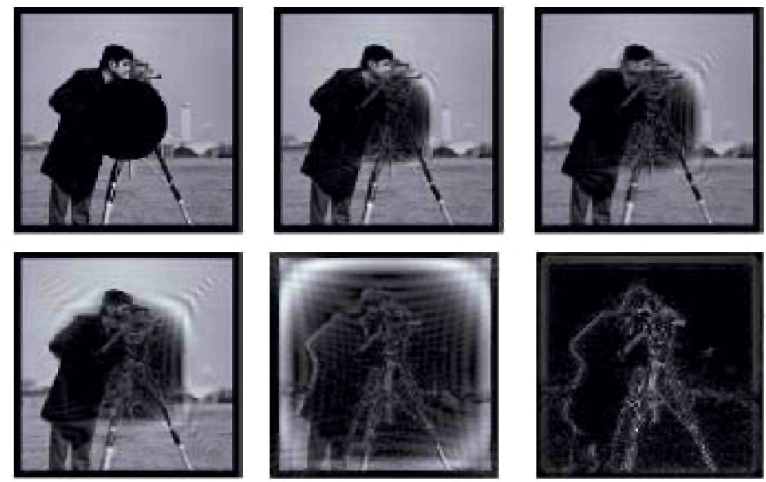

FIGURE 3. H-filtering in the gyrator domain for angles $\alpha=0^{\circ}, 20^{\circ}, 35^{\circ}, 45^{\circ}, 65^{\circ}, 90^{\circ}$ correspondingly.
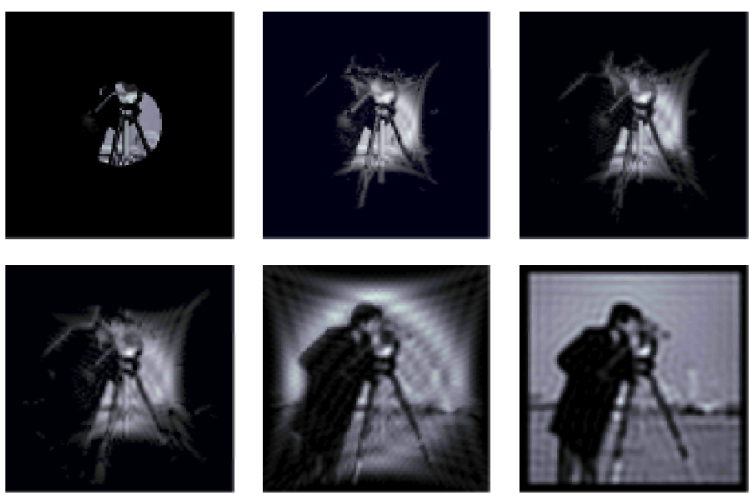

FIGURE 4. L-filtering in the gyrator domain for angles $\alpha=0^{\circ}, 20^{\circ}, 35^{\circ}, 45^{\circ}, 65^{\circ}, 90^{\circ}$ correspondingly. 
Another example is related to the reduction of hyperbolic type noise in the appropriate gyrator domains. As well as the plane waves (or more general periodic noise) are localized in the Fourier domain, the spherical waves (also known as chirp functions) can be localized in the appropriated fractional Fourier domains [2]. From line 2 of the Table 2 it follows that the hyperbolic waves are squeezed to the Dirac $\delta$ function by the corresponding GT. This fact can be used for elimination of the hyperbolic type noise in the gyrator domains. The test image $s$ corrupted by the additive hyperbolic noise

$$
f(x, y)=s(x, y)+A \cos \left(2 \pi c\left(x-v_{x}\right)\left(y-v_{y}\right)\right)
$$

is represented in Fig. 5 (a). Performing its GT at angle $\alpha=\operatorname{arccot}(c)$ we observe a bright spot in the left low comer (Fig. 5 (b)) which corresponds to the noise. Eliminating this point and repeating the procedure for the GT at angle $\alpha=-\operatorname{arccot}(c)$ the noise reduced image is obtained (Fig. 5(c)). In the example $\alpha=10 \pi / 9$. The entire filtering procedure requires performing three gyrator transforms at angles $\alpha,-2 \alpha, \alpha$ and two filtering steps in order to eliminate two exponential noise components.

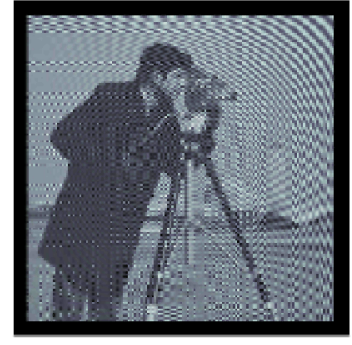

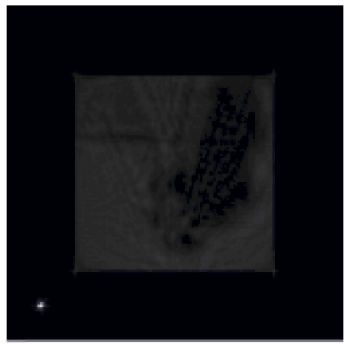

b

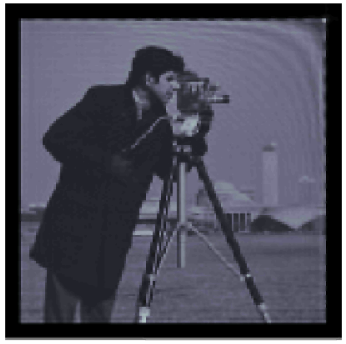

$\mathrm{c}$

FIGURE 5. Image corrupted by the hyperbolic noise (a), Amplitude of the GT of the image (b), Noise reduced image reconstructed after filtering in the corresponding GT domains (c).

As well as the fractional FT $[11,12]$ the GT can be used for information encryption since not only the random phase masks but also the orders of the gyrator domain (angles) where they are located play the role of encryption keys.

\section{CONCLUSIONS}

The representation of the two-dimensional signal in new phase space domains different from the Fourier or fractional Fourier ones opens new perspectives for information processing. We have demonstrated the possible applications of the GT for the image filtering, noise reduction, and encryption. Besides that the GT promises to be a useful tool for beam characterization, quantum information, new mode generation, etc. The existence of the flexible optical set up which permits to change 
easily the parameter $\alpha$ of the GT makes this transform a powerful tool for optical information processing.

\section{ACKNOWLEDGMENTS}

Spanish Ministry of Education and Science is acknowledged for financial support, project TEC 2005-02180/MIC.

\section{REFERENCES}

1. A. Van der Lugt, ed., Optical Signal Processing, John Wiley\&Sons Inc., New York, (1992).

2. H. M. Ozaktas, Z. Zalevsky, and M. Alper Kutay, The Fractional Fourier Transform with Applications in Optics and Signal Processing, John Wiley\&Sons, NY, USA (2001).

3. E. G. Abramochkin and V. G. Volostnikov, "Generalized Gaussian beams," J. Opt. A.: Pure Appl. Opt. 6, S157-S161 (2004).

4. M. W. Beijersbergen, L. Allen, H. E. L. O. Van der Veen, and J. P. Woerdman, "Astigmatic laser mode converters and transfer of orbital angular momentum," Opt. Commun. 96, 123-132 (1993).

5. R. Simon and K. B. Wolf, "Structure of the set of paraxial optical systems," J. Opt. Soc. Am. A 17, $342-355(2000)$.

6. J. A. Rodrigo, T. Alieva, and M. L. Calvo, "Optical system design for ortho-symplectic transformations in phase space," J. Opt. Soc. Am. A 23, 2494-2500 (2006).

7. J. A. Rodrigo, T. Alieva, and M. L. Calvo, "Gyrator transform properties and applications," Opt. Express 15, 2190-2203 (2007).

8. T. Alieva, M.J. Bastiaans, and M. L. Calvo, "Fractional transforms in optical information processing," EURASIP J. Appl. Signal Process. 2005, pp. 1498-1519 (2005).

9. M. Bastiaans and T. Alieva, "First-order optical systems with unimodular eigenvalues," J. Opt. Soc. Am. A 23, 1875-1883 (2006).

10.A. W. Lohmann, Z. Zalevsky, and D. Mendlovic, "Synthesis of pattern recognition filters for fractional Fourier processing," Optics Communications 128, 199-204 (1996).

11. N. Towghi, B. Javidi, Z. Luo, "Fully phase encrypted image processor," J. Opt. Soc. Am. A 16,19151927 (1999).

12. G. Unnikrishnan, J. Joseph, and K. Singh, "Optical encryption by double-random phase encording in the fractional Fourier domain," Opt. Lett. 25, 887-889 (2000). 
Copyright of AIP Conference Proceedings is the property of American Institute of Physics and its content may not be copied or emailed to multiple sites or posted to a listserv without the copyright holder's express written permission. However, users may print, download, or email articles for individual use. 